(1)

CrossMark

\title{
The ageing brain in sleep apnoea: paradoxical resilience, survival of the fittest, or simply comparing apples and oranges?
}

\author{
David Gozal
}

Affiliation: Dept of Pediatrics, Section of Sleep Medicine, Pritzker School of Medicine, Biological Sciences Division, The University of Chicago, Chicago, IL, USA.

Correspondence: David Gozal, Dept of Pediatrics, Section of Sleep Medicine, Pritzker School of Medicine, The University of Chicago, KCBD, Room 4100, 900 E. 57th Street, Mailbox 4, Chicago, IL 60637, USA. E-mail: dgozalduchicago.edu

@ERSpublications

Preserved brain integrity in older sleep apnoea patients depends on whether underlying symptoms are present or not http://ow.ly/O0U530jX1Xp

Cite this article as: Gozal D. The ageing brain in sleep apnoea: paradoxical resilience, survival of the fittest, or simply comparing apples and oranges? Eur Respir J 2018; 51: 1800802 [https://doi.org/10.1183/ 13993003.00802-2018].

The elevated prevalence of obstructive sleep apnoea (OSA) and its association with a long and ever expanding list of end-organ morbidities has prompted substantial interest in identifying mechanistic pathways underlying such deleterious consequences, and their potential reversibility with treatment. Over the last several decades, we have witnessed substantial increases in the granularity of the morbid phenotype of OSA, and such observations have prompted us to rethink some of the stricter early concepts. Among them, rather than viewing OSA as a single disease model that is applicable to all patients, we have formulated the conceptual framework of distinct phenotypes of OSA that may be driven by different mechanisms, link to different clinical manifestations, and display divergent responses to therapy [1-6]. In this contextual setting, the aspirational goals of the field are to first and foremost identify those OSA patients likely to benefit from treatment and administer the correct intervention, i.e. precision medicine [7, 8].

The initial awareness to the importance of excessive daytime sleepiness (EDS) in the therapeutic response [9] spurred subsequent exploration of additional factors influencing clinical aspects of OSA and associated morbidities. Among these, ageing has emerged as an important modifier of OSA disease cardiovascular outcomes [10,11], whereby the odds of significant associations between OSA and end-organ morbidity appear to decline with advancing age beyond 55 years of age [12-15]. Thus, it would seem that a "survival effect" may be operational among a selected group of OSA patients, and becomes particularly discernible after a specific age threshold has been crossed. However, to what extent ageing-related survival is protective across all morbidities of OSA is unclear.

One of the important, yet vastly heterogeneous adverse manifestations of OSA is the increased risk for neurocognitive deficits [16]. It has become apparent that at any level of OSA severity, there will be patients manifesting cognitive morbidity and those who are apparently unaffected. Initial studies in animal models and humans posited that both developing and ageing brains would be uniquely susceptible to the intermittent hypoxia and sleep perturbations that characterise OSA, and manifest as neuronal cell losses 
within neural regions underlying cognitive functions [14, 17-20]. Furthermore, it would be reasonable to anticipate that such OSA-associated cognitive deficits and the rate of their progression would be further exacerbated by the concurrent presence of risk factors such as a known propensity for Alzheimer disease [21]. Under these circumstances, several studies have provided rather robust evidence appearing to support the assumption that OSA accelerates the ageing processes [22], and as such that their co-existence would therefore augment the risk for adverse outcomes, including cognition and central nervous system (CNS) integrity [22-24]. However, not all of the studies have yielded similar conclusions. Indeed, Celle et al. [25] report in this issue of the European Respiratory Journal that when they evaluated a rather extensive cohort of asymptomatic and otherwise healthy elderly subjects, magnetic resonance imaging (MRI) studies did not reveal changes in local brain volumes or in cortical thickness that were significantly associated with polysomnographic measures traditionally used to evaluated OSA severity. Furthermore, there were no significant differences among the overall rather restricted subgroups for those subjects who had OSA and were treated versus those with OSA who were not treated or healthy controls. The authors attributed their findings to several potential factors, namely the more sophisticated image analysis approach consisting of voxel-based morphometry, the recruitment approach based on a healthy ageing general population without a priori evidence of OSA, and the fact that all subjects were essentially asymptomatic and in their vast majority did not manifest any evidence of EDS. Regarding the latter, EDS was evaluated subjectively by CELLE et al. [25] using the Epworth sleepiness scale rather than objectively, a factor that has previously been considered as disabling the ability to detect those patients with OSA who are more susceptible to develop systemic inflammation, a major determinant of cognitive dysfunction [26-28].

So what do we deduce from so much contradictory evidence? First, that the frequency of cognitive deficits and their underlying brain imaging correlates in a general community-based overall healthy population in whom occasional participants have OSA is likely to be substantially reduced when compared to a clinical symptomatic referral population in whom the presence of both subjective and ultimately objective EDS, along with many other end-organ morbidity related manifestations, will likely result in a much higher proportion of cognitively affected individuals, and consequently a much higher probability of finding structural correlates in brain MRI scans. Of note, one of the potential consequences involved in a healthy asymptomatic cohort selection may include the possibility of such cohort being underpowered to detect the small subset of individuals in whom OSA will translate into positive MRI findings. Second, we need to consider that there is likely a larger subset of aged individuals who are more likely to be protected from OSA-induced damage, if indeed the magnitude of oxidative stress and the changes in a large array of other brain properties elicited by OSA are not as prominently recruited when compared to younger patients [29, 30]. Third, and perhaps more importantly, that the presence of other risk factors increasing the propensity for cognitive deficits in the context of OSA is probably a critical determinant of the clinical phenotype and of the underlying CNS pathology among aged patients. Finally, that similar to many other confounders, age is clearly one important factor that needs to be incorporated into the prediction models of risk, cost and benefit in the context of decisions on therapeutic interventions for OSA. However, we will still need to figure out whether biological or chronological age is the correct temporal factor in the decision-making algorithm. Notwithstanding, as we continue to expand the number of phenotypic groups in OSA, and define increasingly better the unique differentiators between one OSA phenotype and the next, it is very likely that future selection of optimal interventional strategies will need to be predicated on combinatorial clinical and biomarker approaches [7, 31,32], and that among the latter, surrogate reporters of senescence will certainly assume a major role. Till then, and in the words of Mark Twain "Age is an issue of mind over matter. If you don't mind, it doesn't matter."

Conflict of interest: None declared.

Support statement: D. Gozal is supported by the Herbert T. Abelson Chair and National Institutes of Health grants HL130984 and NS034939. Funding information for this article has been deposited with the Crossref Funder Registry.

\section{References}

1 Saaresranta T, Hedner J, Bonsignore MR, et al. Clinical phenotypes and comorbidity in European sleep apnoea patients. PLoS One 2016; 11: e0163439.

2 Younes M. Fifty years of physiology in obstructive sleep apnea. Am J Respir Crit Care Med 2017; 196: 954-957.

3 Zinchuk AV, Jeon S, Koo BB, et al. Polysomnographic phenotypes and their cardiovascular implications in obstructive sleep apnoea. Thorax 2018; 73: 472-480.

4 Khalyfa A, Kheirandish-Gozal L, Khalyfa AA, et al. Circulating plasma extracellular microvesicle microRNA cargo and endothelial dysfunction in children with obstructive sleep apnea. Am J Respir Crit Care Med 2016; 194: 1116-1126.

5 Ramos AR, Figueredo P, Shafazand S, et al. Obstructive sleep apnea phenotypes and markers of vascular disease: A review. Front Neurol 2017; 8: 659.

6 Pien GW, Ye L, Keenan BT, et al. Changing faces of obstructive sleep apnea: treatment effects by cluster designation in the Icelandic Sleep Apnea Cohort. Sleep. 2018; 41: 10.1093/sleep/zsx201. 
7 Sánchez-de-la-Torre M, Khalyfa A, Sánchez-de-la-Torre A, et al. Precision medicine in patients with resistant hypertension and obstructive sleep apnea: blood pressure response to continuous positive airway pressure treatment. J Am Coll Cardiol 2015; 66: 1023-1032.

8 Bonsignore MR, Suarez Giron MC, Marrone O, et al. Personalised medicine in sleep respiratory disorders: focus on obstructive sleep apnoea diagnosis and treatment. Eur Respir Rev 2017; 26: 170069.

9 Barbé F, Mayoralas LR, Duran J, et al. Treatment with continuous positive airway pressure is not effective in patients with sleep apnea but no daytime sleepiness. A randomized, controlled trial. Ann Intern Med 2001; 134: $1015-1023$.

10 Lavie L, Lavie P. Ischemic preconditioning as a possible explanation for the age decline relative mortality in sleep apnea. Med Hypotheses 2006; 66: 1069-1073.

11 Lavie P, Lavie L. Unexpected survival advantage in elderly people with moderate sleep apnoea. J Sleep Res 2009; 18: 397-403

12 Johansson P, Alehagen U, Svanborg E, et al. Clinical characteristics and mortality risk in relation to obstructive and central sleep apnoea in community-dwelling elderly individuals: a 7-year follow-up. Age Ageing 2012; 41: $468-474$.

13 Tsapanou A, Gu Y, O'Shea D, et al. Self-reported sleep disordered breathing as risk factor for mortality in the elderly. J Stroke Cerebrovasc Dis 2016; 25: 1524-1531.

14 Terpening Z, Lewis SJ, Yee BJ, et al. Association between sleep-disordered breathing and neuropsychological performance in older adults with mild cognitive impairment. J Alzheimers Dis 2015; 46: 157-165.

15 Duffy SL, Lagopoulos J, Terpening Z, et al. Association of anterior cingulate glutathione with sleep apnea in older adults at-risk for dementia. Sleep 2016; 39: 899-906.

16 Gozal D. CrossTalk proposal: the intermittent hypoxia attending severe obstructive sleep apnoea does lead to alterations in brain structure and function. J Physiol (Lond) 2013; 591: 379-381.

17 Gozal E, Row BW, Schurr A, et al. Developmental differences in cortical and hippocampal vulnerability to intermittent hypoxia in the rat. Neurosci Lett 2001; 305: 197-201.

18 Gozal D, Row BW, Kheirandish L, et al. Increased susceptibility to intermittent hypoxia in aging rats: changes in proteasomal activity, neuronal apoptosis and spatial function. J Neurochem 2003; 86: 1545-1552.

19 Kamba M, Inoue Y, Higami S, et al. Age-related changes in cerebral lactate metabolism in sleep-disordered breathing. Neurobiol Aging 2003; 24: 753-760.

20 Ayalon L, Ancoli-Israel S, Drummond SP. Obstructive sleep apnea and age: a double insult to brain function? Am J Respir Crit Care Med. 2010; 182: 413-419.

21 Baril AA, Gagnon K, Arbour C, et al. Regional cerebral blood flow during wakeful rest in older subjects with mild to severe obstructive sleep apnea. Sleep 2015; 38: 1439-1449.

22 Gaspar LS, Álvaro AR, Moita J, et al. Obstructive sleep apnea and hallmarks of aging. Trends Mol Med 2017; 23: 675-692.

23 Kerner NA, Roose SP, Pelton GH, et al. Association of obstructive sleep apnea with episodic memory and cerebral microvascular pathology: A preliminary study. Am J Geriatr Psychiatry 2017; 25: 316-325.

24 Pereira AC, Mao X, Jiang CS, et al. Dorsolateral prefrontal cortex GABA deficit in older adults with sleep-disordered breathing. Proc Natl Acad Sci USA 2017; 114: 10250-10255.

25 Celle S, Boutet C, Annweiler C, et al. Sleep apnoea in asymptomatic elderly: a real issue for the brain? Eur Respir J 2018; 51: 1702450 .

26 Li Y, Vgontzas AN, Fernandez-Mendoza J, et al. Objective, but not subjective, sleepiness is associated with inflammation in sleep apnea. Sleep 2017; 40: 10.1093/sleep/zsw033.

27 Li RC, Row BW, Gozal E, et al. Cyclooxygenase 2 and intermittent hypoxia-induced spatial deficits in the rat. Am J Respir Crit Care Med 2003; 168: 469-475.

28 Haensel A, Bardwell WA, Mills PJ, et al. Relationship between inflammation and cognitive function in obstructive sleep apnea. Sleep Breath 2009; 13: 35-41.

29 Dalmases M, Torres M, Márquez-Kisinousky L, et al. Brain tissue hypoxia and oxidative stress induced by obstructive apneas is different in young and aged rats. Sleep 2014; 37: 1249-1256.

30 Jorba I, Menal MJ, Torres M, et al. Ageing and chronic intermittent hypoxia mimicking sleep apnea do not modify local brain tissue stiffness in healthy mice. J Mech Behav Biomed Mater 2017; 71: 106-113.

31 De Luca Canto G, Pachêco-Pereira C, Aydinoz S, et al. Biomarkers associated with obstructive sleep apnea and morbidities: a scoping review. Sleep Med 2015; 16: 347-357.

32 Kheirandish-Gozal L, Gozal D. Pediatric OSA syndrome morbidity biomarkers: the hunt is finally on. Chest 2017; 151: 500-506. 